\title{
Microcannular tumescent liposuction
}

\author{
Jayashree Venkataram, Mysore Venkataram* \\ Consultant Gynaecologist and Liposuction Surgeon, *Consultant Dermatologist and Hair Transplant Surgeon, Venkat Charmalaya-Centre for \\ Advanced Dermatology, Bangalore, India
}

Address for correspondence: Dr. Venkataram Mysore, Venkat Charmalaya-Centre for Advanced Dermatology, Bangalore, India. E-mail: mysorevenkat@hotmail.com

\section{ABSTRACT}

Liposuction is a commonly performed procedure to remove localized deposits of fat. Liposuction under general anesthesia is associated with significant morbidity and risk of mortality. Dermatologic surgeons have made significant contributions in this field. Tumescent liposuction using microcannuale under local anesthesia, as practised by dermatologic surgeons is safe and effective. This article describes the procedure of microcannular tumescent liposuction.

Key Words: Liposuction, tumescent anesthesia

\section{INTRODUCTION}

Liposuction is the surgical removal of subcutaneous fat by means of aspiration cannulae introduced through small skin incisions, assisted by suction. Synonyms include liposuction surgery, suction-assisted lipectomy, suction lipoplasty, fat suction, blunt suction lipectomy and liposculpture..$^{[1,2]}$

Liposuction has emerged as one of the most commonly performed cosmetic procedures today. ${ }^{[1]}$ Dermatologists now perform about one third of these procedures in the United States and have pioneered many of the advances in liposuction, especially in the fields of ambulatory surgery and local anesthesia. ${ }^{[1]}$ This article discusses microcannular tumescent liposuction, which has emerged as the gold standard method in liposuction.

\section{STRUCTURE OF FAT ${ }^{[3-6]}$}

Subcutaneous fat is arranged in the form of lobules separated from each other by septa. The fibrous septum consists of blood vessels, nerves and lymphatics. Several lobules together form pearls, which in turn are organized into fat sections and then into fat compartments. Each lobule consists of fat cells, which consist mostly of triglycerides, which fill up the cell almost entirely, pushing the nucleus to one side.
New fat cells are formed from fibroblasts and function as a storage site of energy. Several factors such as diet, exercise, hormones (such as steroids, thyroxin and glucagon) affect fat cells. It has been shown that with initial weight gain in any person, there is an increase in the size of the fat cell. With continued weight gain, there is in addition, an increase in the fat cell number. Diet and exercise have been shown to decrease the fat cell size but not the fat cell number, which is referred to as "resistant fat".

Liposuction is a method of reducing the fat cell number and thereby the resistant fat. Liposuction removes the resistant fat by two mechanisms;

1. Removal of fat cells

2. Damaging the fat cells: The to and fro motion of the cannula damages many of the remaining fat and other fibrous cells. These remaining damaged fat cells get absorbed slowly. Thus, the full results are seen after 6-12 weeks of liposuction [Figure 1].

\section{HISTORY OF LIPOSUCTION ${ }^{[7,8]}$}

Liposuction was initially developed in 1978-1982 in Italy and France. At that time, liposuction could only be performed under general anesthesia and was called "dry liposuction" as no fluid was introduced into fat. Later, a small amount of fluid 
was introduced into fat and this was referred to as the "wet technique". These methods were associated with much blood loss and patients frequently required blood transfusions. In 1985, Dr. Jeffrey A. Klein, M.D., a dermatologist in California, revolutionized liposuction surgery when he developed the tumescent technique, which permits liposuction totally by local anesthesia and virtually eliminates surgical blood loss. ${ }^{[8]}$ The tumescent technique has now become the worldwide standard of care for liposuction..$^{[1,2]}$

\section{INDICATIONS FOR LIPOSUCTION ${ }^{[1,2]}$}

Liposuction can be used in the following situations:

1. Fat, which is resistant to diet or exercise, located in any area of the body, such as the abdomen, thighs, hips, neck, face and under the chin.

2. Inherited localized accumulations of fat.

3. For breast reduction. In men, gynecomastia is an important indication for liposuction.

4. Liposuction has been found useful for noncosmetic indications also - hyperhidrosis of axillae, ${ }^{[9]}$ lipomas. ${ }^{[10]}$

\section{METHODS OF LIPOSUCTION ${ }^{[1,2]}$}

\section{Conventional liposuction}

Conventional liposuction is largely practised by plastic surgeons using large cannulae under general anesthesia. This method is performed as follows:

a) General anesthesia is used.

b) Introduction of small amount of fluid into fat.

c) Making large incisions $(1.5 \mathrm{~cm})$ to introduce cannulae.

d) Sucking out large amounts of fat, often 8-10 litres quickly through large cannulae $6 \mathrm{~mm}-1 \mathrm{~cm}$ in diameter.

e) Suturing the incision wounds of the cannulae.

The whole procedure lasts 2-3 hours. Thus, this method is quick and can remove large amounts of fat. However, it has the following disadvantages:

1. As the method is performed under general anesthesia, the patient has to be hospitalized.

2. Hospitalization adds significantly to the cost.

3. General anesthesia always has its risks.

4. Use of large cannulae causes damage to tissue and hence, increases bleeding. This technique is associated with producing aspirates that are $30-40 \%$ blood by volume, ${ }^{[11,12]}$ often needing blood transfusion.

5. Risk of side effects such as fat embolism.

6. Large cannulae need large incisions, which have to be sutured and heal with significant scars.

7. Recovery time is slow as after any procedure performed under general anesthesia.

\section{Microcannular tumescent liposuction}

Microcannular tumescent liposuction is performed totally by using local anesthesia (called tumescent anesthesia): ${ }^{[9,13]}$ The word "tumescent" means swollen and firm. ${ }^{[8]}$ This technique involves subcutaneous infiltration of large volumes of crystalloid fluid containing low concentrations of lidocaine and epinephrine (called Klein solution) followed by suction-assisted aspiration of fat by using small aspiration cannulae called microcannuale. It is a method for performing liposuction surgery with the patient under local anesthesia. ${ }^{[1,2]}$ The term "tumescent liposuction" specifically excludes the use of any additional anesthesia.

In summary, the procedure of tumescent anesthesia is as follows:

1. Introduction of a large amount (1-4 L) of Klein's solution into fat both for purposes of ballooning the fat tissue so as to decrease bleeding and, for anesthesia.

3. Making several small incisions called 'adits' $(1-3 \mathrm{~mm})$ to introduce microcannulae.

4. Sucking the fat out through small cannulae (microcannuale) $1.5-3 \mathrm{~mm}$ in diameter.

5. Leaving the incision wounds of cannulae open to drain out fluid. A small amount of fluid is left back in tissue and is allowed to drain slowly over two days. This residual fluid provides analgesia in the postoperative period.

6. Applying compression bandages and sending the patient home without any hospitalization.

The whole procedure lasts 3-5 hours. Table 1 shows the comparison between the two methods:

\section{OTHER METHODS OF LIPOSUCTION}

Power-assisted liposuction ${ }^{[14]}$ with a reciprocating cannula is a new technology for liposuction and shows initial promise. In powered liposuction, the reciprocating motion mimics the to-and-fro action of the surgeon's cannula movement, decreasing the work of the procedure and is therefore, less tiring for the physician. In addition, it allows the surgeon to remove fat more completely in "tight" areas where forceful cannula movements are difficult because of physical space constraints (e.g., periumbilical and waist areas). However, if powered cannulae are used to suck larger amounts of fat, they can only be beneficial with cannulae that are $\geq 3.5 \mathrm{~mm}$ in diameter, which cause significantly more pain. Usually powerassisted liposuction also needs concomitant intramuscular (IM) or intravenous (IV) narcotics and sedatives as well as nitrous oxide sometimes. These features in turn negate the above-mentioned advantages of tumescent liposuction. 
Table 1: Comparison of techniques of liposuction

\begin{tabular}{lll}
\hline & Conventional method & Tumescent method \\
\hline Pain control & General anesthesia & Local anesthesia \\
Risks & Higher complication rate & Lower complication rate \\
Bleeding & Significant blood loss often requiring transfusion & Minimal blood loss \\
Postoperative period & No long-lasting pain relief. Requires strong, & Pain relief up to 24 hours, only minimal oral \\
& parenteral pain killers & pain killers enough \\
Hospital admission & Required & Outpatient procedure \\
Return to work & Days to weeks & $1-4$ days \\
Cannula size & Larger cannulae & Smaller cannulae \\
Sutures & Required & Not required \\
Speed & Quick & Slow \\
Amount of fat & Large, 8-10 liters & Smaller, 2-5 liters \\
Cost & Expensive because of hospital stay & Less expensive
\end{tabular}

Ultrasound-assisted liposuction (UAL) ${ }^{[15]}$ was introduced to damage the fat cells and thereby facilitate the removal of fat. However, the method had significant side effects such as burns of the skin. The damaged fat also led to small cysts containing fluid called seromas. Ultrasonic-assisted liposuction is associated with significant bruising and prolonged postoperative swelling. Most importantly, the ultrasound machines are expensive, increasing the cost of the procedure.

Principle of tumescent anesthesia ${ }^{[16-[20]}$ : The most important aspect of tumescent liposuction is that a local anesthetic is used over a wide area to provide anesthesia and analgesia, using sufficient quantity of lignocaine far in excess of the conventional dosage. The underlying principle of tumescent anesthesia is that contrary to conventional thinking, larger doses of lidocaine, even up to $45-55 \mathrm{mg} / \mathrm{kg}$ weight can be administered safely. Conventional teaching has widely regarded, without adequate pharmacological proof, that the safe upper limit for lignocaine administration is $6 \mathrm{mg} /$ $\mathrm{kg}$ body weight. In a radical departure from this hitherto accepted fact, Klein showed that in tumescent anesthesia, much higher doses can safely be administered because the rate of absorption of lignocaine is slow, leading to smaller peak values and hence, lesser toxicity. The reasons for the slow absorption of lignocaine are:

i) Subcutaneous fat has a low volume of blood flow.

ii) Lignocaine is lipophilic and is easily sequestered in fat.

iii) Diluted epinephrine in saline solution ensures vasoconstriction, thus minimizing systemic absorption and bleeding.

iv) The large volume of tumescent solution itself compresses blood vessels by hydrostatic pressure.

V) The very low dilution of lignocaine in Klein's solution does not achieve the gradient required for systemic absorption.

vi) Most of the solution is removed during aspiration, minimizing the duration available for absorption.
The usual tumescent solution concentration used by dermatologic surgeons is $0.05-0.1 \%$ lidocaine. The concentration of epinephrine is at $1: 1,000,000$ to $1.5: 1,000,000$. Approximately 10 meq. of sodium bicarbonate is added to one litre of tumescent solution to raise the $\mathrm{pH}$ and to prevent stinging due to the acidic $\mathrm{pH}$ of lidocaine. The required lidocaine dosages are dependent on appropriate epinephrine concentration in the tumescent solution. The recommended maximum dose of lidocaine is $55 \mathrm{mg} / \mathrm{kg}$ for most patients. The recommended concentration of epinephrine in tumescent solutions is $0.25-1.5 \mathrm{mg} / \mathrm{L}$. The total dosage of epinephrine should be minimized within these limits and usually should not exceed $50 \mu \mathrm{g} / \mathrm{kg}$. Since lidocaine is metabolized by the liver, drugs that compete with the cytochrome $\mathrm{P}_{450}$ enzyme system that or displace lidocaine from plasma proteins can increase lidocaine blood levels and cause lidocaine toxicity. The physician must be aware of these drug interactions when planning anesthesia for the patient.

\section{PROCEDURE OF TUMESCENT LIPOSUCTION}

Patient selection ${ }^{[1,2,5,17,21]}$; Proper patient selection is highly important-the best candidates are patients with localized deposits of fat, who are not grossly obese, without significant medical problems and have realistic expectations of what liposuction can accomplish. There is no definite age or weight limit for patients who are "good candidates" for liposuction.

The maximum amount of fat that can be removed safely by tumescent liposuction is probably about 3-5 liters. ${ }^{10]}$ Generally, it is advisable not to be very aggressive (the socalled megaliposuctions) to avoid complications ${ }^{[1,2]}$. The risk of side effects increases with removal of greater amounts of fat. Different areas such as the abdomen and the thigh or the buttock are not performed together in one session. ${ }^{[18]}$ However, it is possible to treat both buttocks or both thighs in one session. If a patient desires more than one area or 


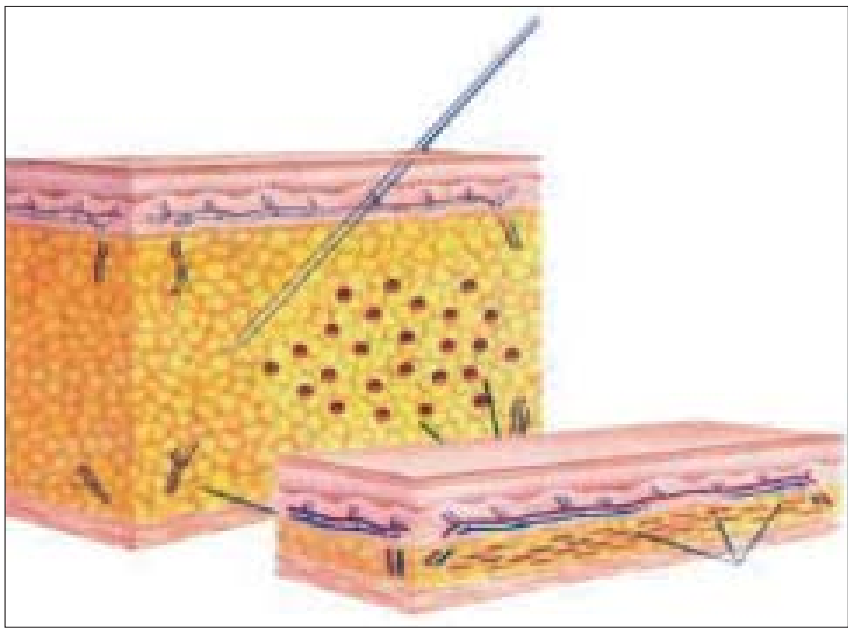

Figure 1: Principle of liposuction; fat before and after aspiration

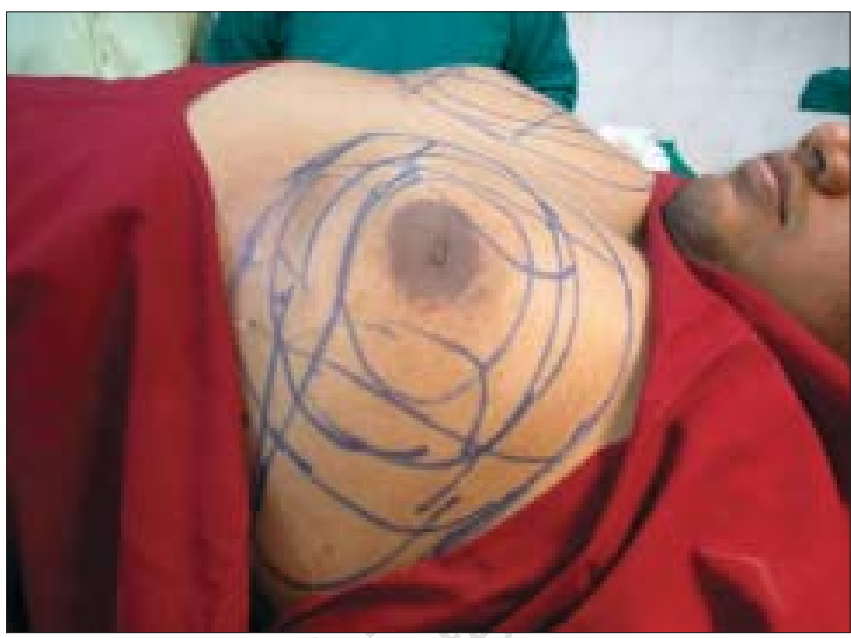

Figure 2: Topographic marking
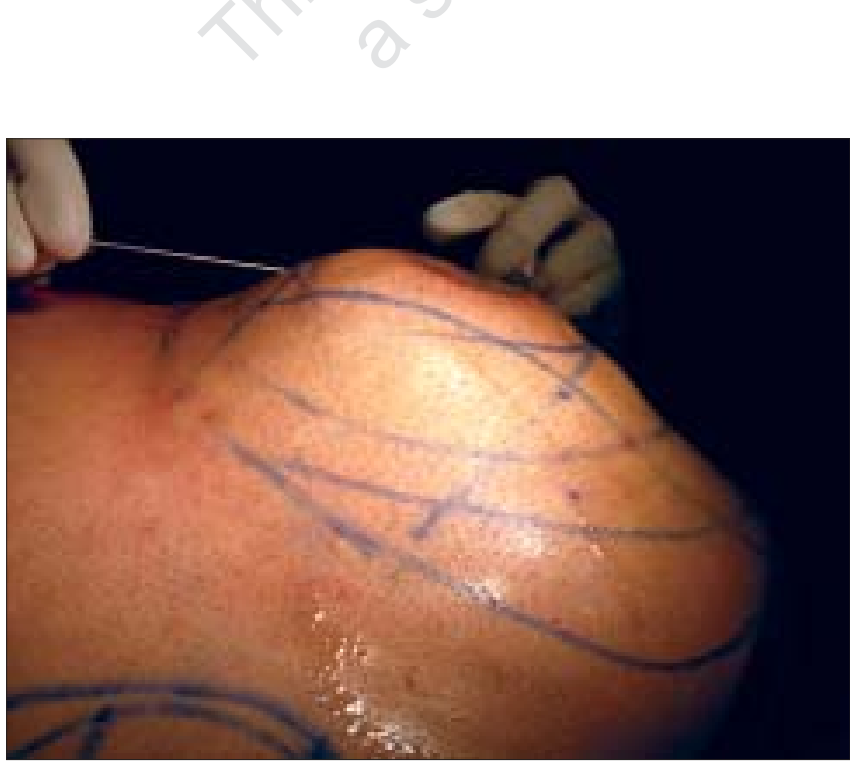

Figure 3: Infiltration of tumescent fluid

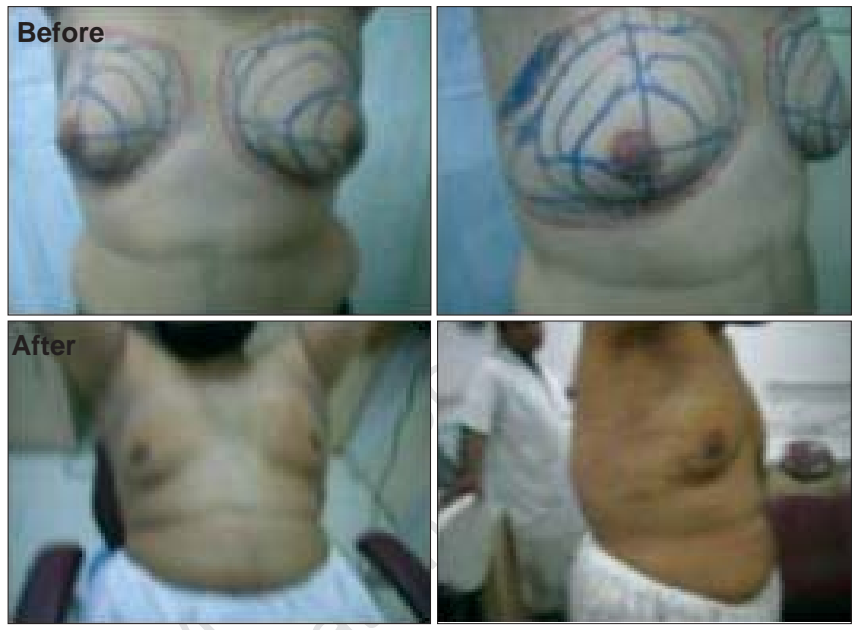

Figure 4: Liposuction results

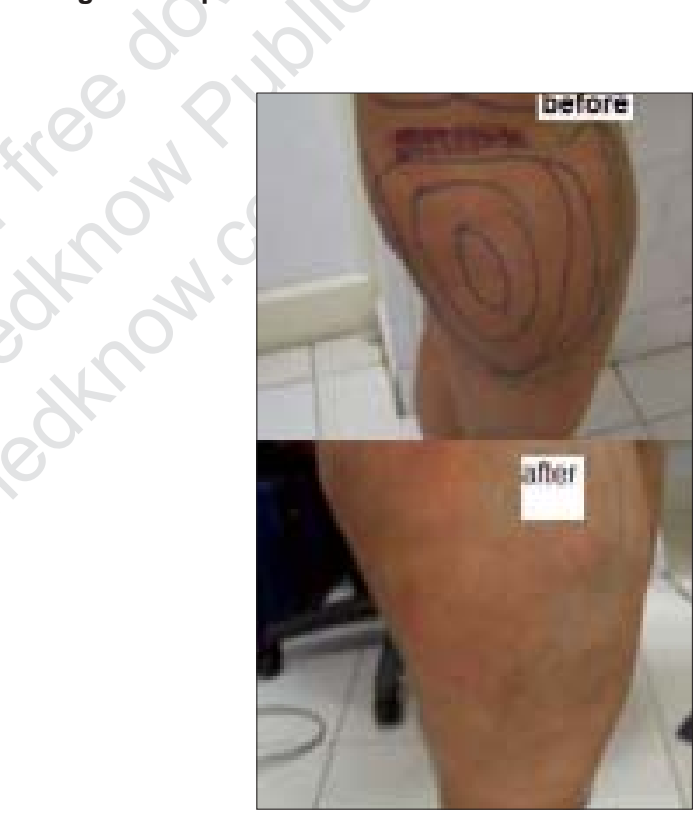

Figure 5: Liposuction results

needs more than 3-5 liters of fat removal, the procedure can be repeated any time after two weeks.

A thorough medical history with particular reference to history of bleeding diathesis, emboli, thrombophlebitis, infectious diseases, poor wound healing and diabetes mellitus is taken. Patients with a medical history of these conditions should receive physician examination and clearance before undergoing liposuction. The history should also include prior abdominal surgeries such as cesarean sections, which produce scarring.

The physician must perform a detailed physical examination to determine that the areas of interest indicated by the patient are amenable to liposuction. The dermatologic surgeon must 
give due consideration to the patient's motivation in seeking liposuction and the patient's expectations of the result.

Liposuction is contraindicated in patients with severe cardiovascular disease, severe coagulation disorders including thrombophilia and during pregnancy.

Counseling: Counseling should include:

a) Discussion on different management options including the role of diet and exercise.

b) Detailed explanation about the surgical procedure including possible postoperative complications.

c) Specific instructions that full results would be seen after 6-12 weeks.

d) Instruction that although the fat removed by liposuction normally does not come back, there may be recurrence if the patient puts on excessive weight. Hence, the importance of continued exercise and diet regulation should be stressed.

e) Any allergies or medical condition that the patient may have should be recorded.

f) As in any cosmetic procedure, the patient should not expect to achieve perfection.

g) Patients should be told not to expect dramatic weight loss with liposuction. Weight lost is equal only to the amount of fat removed-about 3-5 kg.

h) Patients should also understand that while liposuction improves the silhouette of the body, it does not necessarily eliminate cellulite or striae. The patient can also be assured that there is no likelihood of loose skin hanging in the operative area due to the elasticity of skin. A small to moderate amount of excess skin retracts after liposuction even in patients with less than optimal skin elasticity (older than 40 years). Abdominoplasty is usually not necessary for abdominal contouring. It is only necessary if a large amount of excess skin or muscle laxity is present. ${ }^{\mid 22,23]}$

Preoperative instructions: ${ }^{[21]}$ These are routine and include:

a) Routine blood investigations such as blood counts, bleeding and clotting time, blood sugar, hepatitis B surface antigen (HbsAg), human immunodeficiency virus (HIV)-enzyme-linked immunosorbent assay (ELISA) and electrocardiogram (ECG) and liver function tests.

b) Advice to stop smoking as smoking increases intraoperative bleeding

c) Avoidance of oral nonsteroidal anti-inflammatory drugs (NSAIDs) as these may increase intraoperative bleeding

d) Preoperative tranquilizers such as diazepam or lorazepam on the night before surgery

e) Injection vitamin $\mathrm{K}$ to minimize postoperative bruising

\section{Preparation of patient:}

a) Preoperative antibiotic such as cephalexin.

b) Preoperative tranquilizer such as oral lorazepam $1 \mathrm{mg}$.

c) Oral clonidine $0.1 \mathrm{mg}$ to prevent epinephrine-induced tachycardia and as an adjuvant anxiolytic drug.

d) Surgical cleaning of the donor area with povidone iodine.

e) The area for liposuction is topographically marked with marker ink to delineate the bulges and asymmetry [Figure 2].

f) Monitoring: Baseline vital signs including blood pressure and heart rate, are to be recorded preoperatively and postoperatively. Pulse oximeter monitoring is essential. Medical personnel trained in resuscitation should be available on the premises.

Tumescent anesthesia: This is a very important and vital step. Proper tumescence will ensure painless and smooth aspiration.

a) Adits are small holes made for insertion of the infiltration cannulae. These are done with 1.5-2 mm dermal punches at different locations of the area under infiltration anesthesia with $1 \mathrm{ml}$ of $2 \%$ lignocaine. The number of adits needed depends on the area involved. Normally about $6-8$ adits are needed for the abdomen.

b) Tumescent fluid is prepared as discussed earlier.

c) Infiltration of tumescent fluid [Figure 3]: The delivery system for the tumescent solution consists of infusion bags, infiltration pressure cuffs or an infiltration pump to hasten delivery of the fluid and infiltration cannulae of size 0.5-1 mm. About 2-3 litres of fluid are infiltereded gradually in different directions, first into deeper layers of fat and then into the superficial layers. The end point is a firm feel of the skin, which makes the skin swollen and difficult to grasp. It is important to be slow and to avoid jerky, sudden movements to avoid pain. Normally it takes about 1-2 hours for proper anesthesia.

d) Detumescence: It is important to wait for about 30 minutes after tumescence for the infiltration fluid to percolate properly and its full pharmacological effects to take effect. This is indicated by the slight decrease in firmness and the ability to grasp the skin.

\section{Aspiration $^{[24,25]}$}

The most important aspect of proper aspiration is the slow, repeated, to and fro movements of the cannulae. The cannulae are of different sizes varying in diameter from 1-2.5 
$\mathrm{mm}$. Cannulae larger than $4.5 \mathrm{~mm}$ in diameter are not used. A smaller cannula $(1 \mathrm{~mm})$ is first used to create tunnels in the fat. Cannulae of gradually increasing diameter are then employed to aspirate fat. It should be noted that smaller cannulae are equally efficient in aspirating fat as large cannulae and cause less trauma and pain although more time-consuming. Patience is of the essence and the urge to be quick and aggressive is to be discouraged. Deeper layers of fat are aspirated first and then the superficial layers. The movement of the handle is to and fro, slowly in a rhythmic fashion in a fan-like pattern in different directions. Sudden, jerky movements should be avoided. The direction of the handles is always at an angle to the skin and is never vertical. The nonoperating hand is used as a guide to push the fat in the direction of aspiration and also to feel the tip of the cannula to prevent damage to the overlying skin or underlying structures. It is also important to avoid skin trauma at the adit to ensure proper healing of the adits. Care should be exercised to prevent too much aspiration from a given area to avoid dimpling and asymmetry. Different areas are aspirated and then compared for symmetry and regularity. One great advantage of tumescent anesthesia is because the patient is conscious, he / she will feel the pain and warn the surgeon if the cannula is moved deep into muscle or into the surrounding unanesthetized area. Also, he / she is in a position to stand so that the surgeon can compare the two sides for symmetry. The process of aspiration normally takes between 90 minutes to 2 hours and about 2-5 litres of fat are aspirated. Blood loss is minimal and does not exceed $30-50 \mathrm{ml}$ if tumescence is proper. It is important to keep the patient engaged by having a television or music in the theater.

Postoperative dressing and follow-up ${ }^{[26,27]}$ : Postoperative dressing is a very important step in tumescent liposuction. An important feature of the tumescent procedure is that some amount of the fluid is still left behind at the end of the procedure, which ensures anesthesia in the immediate postoperative period thus minimizing the need for potent oral analgesics. This fluid drains out in 3-5 days; for this purpose, the adits are not sutured and are allowed to heal by secondary intention. Tight-pressure bandages are essential to ensure proper drainage of the tumescent fluid. Two layers of pressure dressing (called bimodal compression) are applied to ensure tight compression in the first two days. Dressings are removed on the first postoperative day and the adits are opened again, if necessary, to ensure proper drainage. Improper drainage increases the possibility of panniculitis, secondary infection and irregularity. The pressure in the dressing is decreased after three days and continued for a minimum of two weeks. The patient is advised to come for follow-up for dressing daily for three days. Full results are seen by six weeks [Figures 4-5]. It is important to note that while the patient can return to normal sedentary work in 1-2 days, exercise and undue exertion should be avoided for at least ten days.

Complications ${ }^{[17,28,29 \mid}$ : Tumescent anesthesia is a remarkably safe procedure if all the essential steps are adhered to. In the authors' experience of nearly 200 cases, side effects have been rare.

a) Postoperative pain: This is minimal in the first two days because of the persistent anesthetic fluid in the tissue. Oral analgesics such as paracetamol and infrequently tramadol are all that are required. Mild tenderness at the site of the adits may be felt over 3-5 days. An antibiotic ointment (such as fucidic acid or mupirocin) may be prescribed for application at the site of the adits.

b) Postoperative syncope is a common complication on the first postoperative day. It is vasovagal in origin and is due to the sudden release of pressure while removing the tight bandages. This can be avoided by removing the bandage in supine position and then having the patient sitting in bed for a few minutes before getting up.

c) Postoperative edema is due to the inflammation caused by the aspiration movements and is minimized by using small cannulae and proper postoperative dressings.

d) Postoperative ecchymoses may occur which usually disappear spontaneously over a week. This is common in hypertensive patients and hence, proper blood pressure control is essential.

e) Diffuse tenderness and induration can occur if the drainage is improper.

f) Panniculitis and fat necrosis are rare but they may occur in diabetics. Hence, it is important to ensure proper diabetic control.

g) Postoperative infection is rare if proper aseptic precautions are followed.

h) Seroma formation: Seromas are cystic swellings, which occur due to aggressive superficial fat aspiration. They are more common in ultrasound-assisted aspiration.

i) Irregularity and asymmetry can occur if the amount of fat aspirated is different in different areas and if pressure garments are not worn properly.

Several serious complications have been reported with conventional liposuction, particularly when liposuction is combined with other procedures such as abdominoplasty, the treatment of more than one areas or megaliposuctions (large amounts of fat ( $>5$ litres) are aspirated). These include 
pulmonary embolism, excessive blood loss, hemorrhagic necrosis of fat and even death. ${ }^{[28,29]}$ However, these complications are extremely rare in tumescent liposuction. It is important to note that while mortality has been reported with conventional liposuction, not a single death has been recorded after tumescent liposuction. ${ }^{[1,2,17,29]}$

\section{THE ART OF LIPOSUCTION}

Tumescent liposuction, as a surgical procedure, requires a combination of; (1) practical application of pharmacological knowledge, 2) appreciation of beauty, (3) a perfection of workmanship and (4) skill attained through proper training and clinical experience. In tumescent liposuction, speed and volume of aspirate are not the criteria for excellence. The criteria for excellence are safety, patient comfort, finesse and quality of results. It is important to keep in mind that, as in any cosmetic procedure including liposuction, a final safe and satisfactory result is far more important than quick results.

\section{REFERENCES}

1. Coleman WP, Glogau RG, Klein JA, Moy RL, Guidelines of care for liposuction. J Am Acad Dermatol 2001;45:438-47.

2. Naomi Lawrence, William P. Coleman Liposuction 2002:47:1;105-8.

3. Johnson D, Cormack GC, Abrahams PH, Dixon AK. Computed topographic observations on subcutaneous fat: implications for liposuction. Plast Reconstr Surg 1996;97:387-96.

4. Skouge JW. The biochemistry and development of adipose tissue and pathophysiology of obesity as it relates to liposuction surgery. Dermatol Clin 1990;8:385-93.

5. Lawrence N, Coleman WP III. Liposuction. Adv Dermatol 1996;11:19-49.

6. Klein JA. subcutaneous fat;anatomy and histology In: Klein JA, editor. Tumescent technique. St L ouis: Mosby; 2000. 213-221.

7. Coleman WP III. The history of liposuction and fat transplantation in America. Dermatol Clin 1999;17:723-7.

8. Klein JA: The tumescent technique for liposuction surgery AM J Cosmetic surg 1987 4;1124-1132

9. Lillis PJ, Coleman WP. Liposuction for treatment of axillary hyperhidrosis. Dermatol Clin1990;8:479-82.

10. Pinski KS, Roenigk HH. Liposuction of lipomas. Dermatol Clin
1990;8:483-92.

11. Courtiss EH, Choucair RJ, Donelan MB. Large-volume suction lipectomy: an analysis of 108 patients. Plast Reconstr Surg. 1992;89:1068-1079

12. Mandel MA. Blood and fluid replacement in major liposuction procedures. Aesthetic Plast Surg. 1990;14:187-191

13. Klein JA. Tumescent technique for local anesthesia improves safety in large-volume liposuction. Plast Reconstr Surg. 1993;92:1085-1098

14. Coleman WP. Powered liposuction. Dermatol Surg 2000;26:315-8.

15. Narins R, Coleman WP III, Brody H, Goldman M, Hanke CW, Lillis PJ, et al. Statement on ultrasonic liposuction. Task Force on Ultrasonic Liposuction of the American Society for Dermatologic Surgery. Dermatol Surg 1998;24:1035.

16. Klein JA. Anesthetic formulation of tumescent solutions. Dermatol Clin 1999;17:751-9.

17. Klein J. Two standards of care for tumescent liposuction. Dermatol Surg 1997;23:1194-5

18. Klein JA. Clinical pharmacology. In: Klein JA, editor. Tumescent technique. St Louis: Mosby; 2000. 121-209.

19. Lillis PJ. Liposuction surgery under local anesthesia: limited blood loss and minimal lidocaine absorption. J Dermatol Surg Oncol. 1988;14:1145-1148.

20. Ostad A, Kageyama N, Moy RL. Tumescent anesthesia with a lidocaine dose of $55 \mathrm{mg} / \mathrm{kg}$ is safe for liposuction. Dermatol Surg. 1996;22:921-927

21. Flynn TC, Narins RS. Preoperative evaluation of the liposuction patient. Dermatol Clin 1999;17:729-34.

22. Bank DE, Perez MI. Skin retraction after liposuction in patients over the age of 40. Dermatol Surg 1999;25:673-6.

23. Matarasso A, Matarasso SL. When does your liposuction patient require an abdominoplasty? Dermatol Surg 1997;23:1151-60.

24. Klein JA. Microcannulas. In: Klein JA, editor. Tumescent technique. St Louis: Mosby; 2000. 235-48.

25. Bernstein G. Instrumentation for liposuction. Dermatol Clin 1999; 17:735-49.

26. Klein JA. Post-liposuction care: open drainage and bimodal compression. In: Klein JA, editor. Tumescent technique. St Louis: Mosby; 2000. 281-93.

27. Klein JA. Post-tumescent liposuction care: open drainage and bimodal compression. Dermatol Clin 1999;17:881-90.

28. Rao RB, Ely SF, Hoffman RS. Deaths related to liposuction. N Engl J Med. 1999;340:1471-1475

29. Hanke CW, Bernstein G, Bullock S. Safety of tumescent liposuction in 15,336 patients: national survey results. Dermatol Surg 1995;21;459-62 